\title{
Direct UV-Written Integrated Optical Beam Combiner for Stellar Interferometry
}

\author{
Olivero, Massimo; Svalgaard, Mikael; Jocou, L.; Berger, J.P.
}

Published in:

Journal of Lightwave Technology

Link to article, DOI:

10.1109/JLT.2006.886669

Publication date:

2007

Document Version

Publisher's PDF, also known as Version of record

Link back to DTU Orbit

Citation (APA):

Olivero, M., Svalgaard, M., Jocou, L., \& Berger, J. P. (2007). Direct UV-Written Integrated Optical Beam Combiner for Stellar Interferometry. Journal of Lightwave Technology, 25(1), 367-371.

https://doi.org/10.1109/JLT.2006.886669

\section{General rights}

Copyright and moral rights for the publications made accessible in the public portal are retained by the authors and/or other copyright owners and it is a condition of accessing publications that users recognise and abide by the legal requirements associated with these rights.

- Users may download and print one copy of any publication from the public portal for the purpose of private study or research.

- You may not further distribute the material or use it for any profit-making activity or commercial gain

- You may freely distribute the URL identifying the publication in the public portal 


\title{
Direct UV-Written Integrated Optical Beam Combiner for Stellar Interferometry
}

\author{
Massimo Olivero, Mikael Svalgaard, Laurent Jocou, and Jean-Philippe Berger
}

\begin{abstract}
In this paper, we report the fabrication of an opticalbeam combiner for stellar interferometry by means of direct ultraviolet (UV) writing. The component is shown to have good performance (fringe contrast $>95 \%$, total loss $\sim 0.7,-40-\mathrm{dB}$ crosstalk, broadband operation covering at least the range 1.49-1.65 $\mu \mathrm{m}$, and low differential chromatic dispersion). The overall performance exceeds that of similar components currently used for astronomical research. This result, combined with the fast-prototyping ability of UV writing, opens up new possibilities for the realization of highly optimized integrated-optical components for astronomical research.
\end{abstract}

Index Terms-Astronomy, direct ultraviolet (UV) writing, optical interferometry, optical-device fabrication, optical-planarwaveguide components.

\section{INTRODUCTION}

$\mathbf{O}$ PTICAL stellar interferometers have become fruitful systems for astronomical research due to their capability to overcome the diffraction-limited resolution of individual telescopes and achieve milli-arc-second resolution, thus enabling new endeavors in fields such as imaging of stellar surfaces, study of protoplanetary discs, and active galactic nuclei. Future campaigns for studies of exosolar planets will also use interferometric-measurement techniques.

These systems rely on aperture synthesis interferometry, where light from several telescopes is coherently combined and analyzed. In such a configuration, the angular resolution achieved depends on the telescopes' baseline separation rather than the aperture of each element [1].

The ability to accurately measure the interferometric observables (fringe contrast and phase) has a direct impact on the final quality of the astronomical measurement (e.g., image dynamic range), and this sets stringent requirements for the light-combining system. Beam combination was initially demonstrated using bulk-optical combiners; however, when several telescopes are used for aperture synthesis, the bulk-optical approach becomes very demanding to realize with sufficient stability.

Integrated-optical (IO) technologies have been identified as a new instrumental key that enables to combine a great number

Manuscript received May 25, 2006; revised September 11, 2006.

M. Olivero was with the Research Center COM, Technical University of Denmark, 2800 Lyngby, Denmark. He is now with PhotonLab, Politecnico di Torino, 10129 Torino, Italy (e-mail: massimo.olivero@polito.it).

M. Svalgaard is with the Research Center COM, Technical University of Denmark, 2800 Lyngby, Denmark (e-mail: svlgrd@com.dtu.dk).

L. Jocou and J.-P. Berger are with the Laboratoire d'Astrophysique, Observatoire de Grenoble, 38041 Grenoble Cedex 9, France (e-mail: jocou@ obs.ujf-grenoble.fr; Jean-Philippe.berger@obs.ujf-grenoble.fr).

Digital Object Identifier 10.1109/JLT.2006.886669 of telescopes in a single chip and introduces several advantages which act directly on the instrument accuracy. The IO approach features high inherent stability due to compactness, precise selfalignment, effective modal filtering, and the ability to realize efficient beam-combination schemes [2]. Recently, astronomers have shown that IO components have a good potential for multitelescope aperture synthesis [3]. The components used for that work were fabricated using photolithography and ion exchange [2] or etching [4] processes, which are fairly slow and expensive techniques. Future applications will require stringent performance criteria necessitating extensive device optimization through a closed-loop design process; thus, faster and cheaper prototyping methods are greatly desirable.

Direct UV writing [5] is a fabrication technique, where highperformance waveguides are written directly into a planar glass sample with a focused UV laser beam; hence, it does not require photolithography and is well suited for rapid prototyping. The technology was originally developed for low-cost telecom applications such as variable optical attenuators [6]. Recently, UV writing has been employed for fabrication of devices with increased complexity, such as multichannel optical power splitters [7], [8] and broadband directional couplers [9].

In this paper, we report the first realization of a UVwritten dual-beam combiner for near-infrared stellar interferometry. The demonstrated component features a performance that matches or exceeds that previously achieved with more time-consuming fabrication techniques. Direct UV writing is shown to be a promising technique for realization of highly customizable IO devices for astronomical applications.

\section{Device FAbrication}

The samples used for UV writing are silica-on-silicon threelayer index-matched structures with a $5.4-\mu \mathrm{m}$-thick $\mathrm{Ge} / \mathrm{B}$ doped core layer [10], fabricated by plasma-enhanced chemical vapor deposition (PECVD). The core-layer photosensitivity is enhanced by deuterium loading at 500 bar until saturation prior to UV writing [11]. Channel waveguides are written into the core layer by scanning the sample under a continuous-wave focused 257-nm beam using high-precision translation stages with closed-loop position feedback [9].

The incident UV power is $45 \mathrm{~mW}$ and the desired index step and waveguide width are selected by applying scan velocities in the range 100-900 $\mu \mathrm{m} / \mathrm{s}$. Most of the layout is written at $280 \mu \mathrm{m} / \mathrm{s}$, yielding a waveguide width of $6.1 \mu \mathrm{m}$ and an index step of 0.014. After UV writing, the sample is annealed in a two-step process to first outdiffuse residual deuterium $\left(80^{\circ} \mathrm{C}\right.$, $12 \mathrm{~h})$ and then to reduce the index step to $0.0085\left(320^{\circ} \mathrm{C}\right.$, 


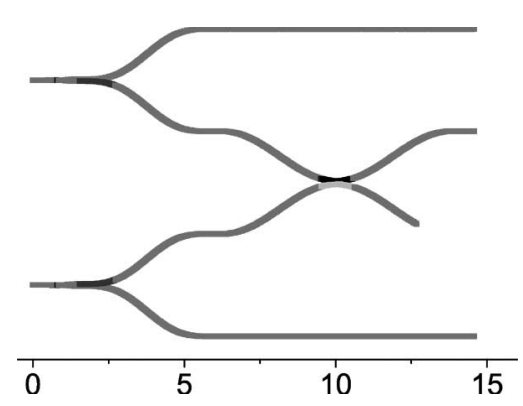

Fig. 1. Layout of UV-written astronomical beam combiner. Dual-beam input: A, B; interferometric output: D, E; photometric output: C, F. Gray shades represent the applied scan velocities in the various sections of the component.

$3 \mathrm{~h}$ ), which enables single-mode operation down to a wavelength of $1.3 \mu \mathrm{m}$. In addition, the high-temperature annealing ensures a high degree of long-term stability of the induced index change [12].

\section{BEAM-COMBINER LAYOUT}

Beam combination for astronomical interferometry places stringent demands on the instrument performance for high light sensitivity and detection of fast fringe changes:

1) low loss (total throughput $>80 \%$ ), to enable high signalto-noise measurements;

2) high-fringe contrast ( $>90 \%$ ), for a high instrumental response (i.e., extended ability to measure pointlike sources);

3) broadband performance (hundreds of nanometers), in order to detect fringes over one or more astronomical photometric bands.

For this demonstration, we have chosen to realize a dual-beam combiner with two interferometric and two photometric outputs (Fig. 1), based on a layout similar to that currently used for scientific measurements in astronomical facilities [3], [13]. Beam interference (port D and E) is achieved with an asymmetrical 3-dB directional coupler [9] that features broadband performance, while the photometric outputs (port $\mathrm{C}$ and F) are realized using 3-dB Y-branch power splitters [7]. The performance of these subcomponents is controlled by tailoring the applied scan velocity during UV writing. Achieving symmetrical Y-branch splitters requires compensation of a proximity effect associated with UV writing [14], which is done by reducing the scan velocity in one of the output arms to $200 \mu \mathrm{m} / \mathrm{s}$. The coupler section follows an asymmetric design to achieve a large bandwidth. Based on previous studies, we have applied scan velocities of $900 / 100 \mu \mathrm{m} / \mathrm{s}$ in the lower/upper arm of the central coupling region to achieve a $3-\mathrm{dB}$ wavelength flattenedcoupling ratio response [9].

A total of 36 beam combiners were fabricated on the same chip, each with a slightly different length of the coupling region so that devices that had a coupling ratio close to the required $3 \mathrm{~dB}$ could later be selected. The input/output-port spacing was set to $500 / 250 \mu \mathrm{m}$ to accommodate commercially available fiber-connector arrays. The total device length is $15 \mathrm{~mm}$, which is $\sim 1 / 3$ of the length of a previous demonstration made by ionexchange technology [2], and each component took just $270 \mathrm{~s}$ to write. The total time required for design, layout definition, and

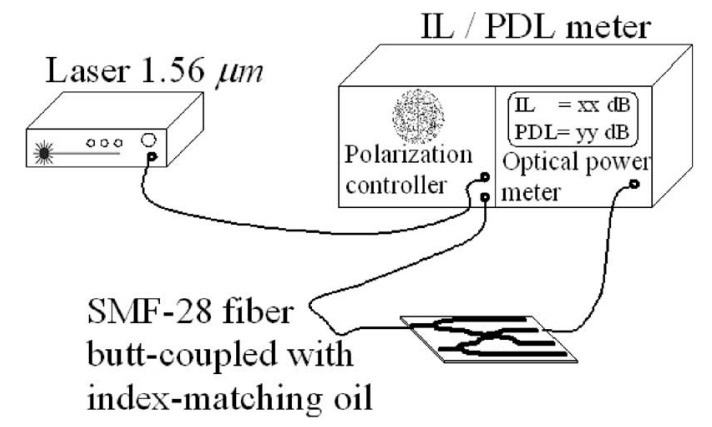

(a)

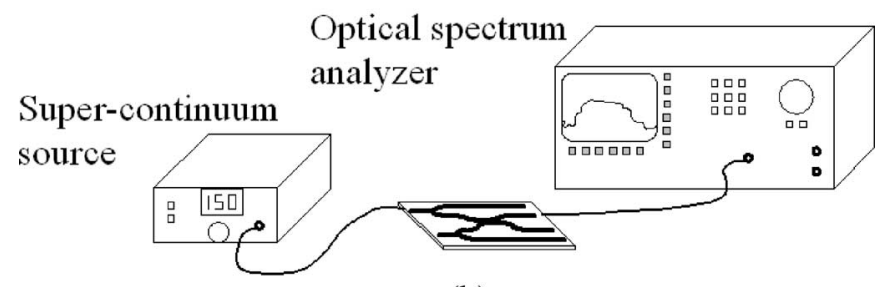

(b)

Fig. 2. Setup for characterization of loss and broadband performance. (a) Measurement of the main parameters (loss, splitting, and coupling ratio) and their polarization dependence with a laser at $1.56 \mu \mathrm{m}$ and an IL/PDL meter. (b) Measurement of broadband response with a supercontinuum source and an optical spectrum analyzer.

fabrication of the component was approximately one week, thus demonstrating the fast-prototyping capability of UV writing.

\section{LOSS PERformance}

The loss and broadband performance was measured using butt-coupled SMF-28 fibers with index matching oil. For excitation, a polarized $1.56-\mu \mathrm{m}$ source or an unpolarized-supercontinuum source was used. In the former case, measurements were performed with an insertion-loss (IL)/polarization-dependent-loss (PDL) meter, while in the latter case, an optical spectrum analyzer was used (Fig. 2). At $1.56 \mu \mathrm{m}$, the PDL was below $0.5 \mathrm{~dB}$ on all output ports while the total transmission loss (sum of all outputs normalized to input signal) was $\sim 0.7 \mathrm{~dB}$ (corresponding to a throughput of $\sim 85 \%$ ), including fiber-waveguide coupling, propagation, bends, etc. The measured loss is essentially due to the bends, since 36-mm-long straight waveguides written on the same chip showed a total IL of $0.3 \mathrm{~dB}$ with PDL at our setup detection limit of $0.2 \mathrm{~dB}$. In earlier experiments, we have estimated the contribution of fiber-waveguide coupling loss by writing waveguides with decreasing lengths and found that this is $\sim 0.03 \mathrm{~dB} /$ facet [8]. Commercial software has also been used to model the overlap integral between the fiber/waveguide modes, yielding a similar result.

Broadband measurements of total transmission loss, splitting ratio, and coupling ratio are presented in Fig. 3. The total loss remains flat at $\sim 0.7 \mathrm{~dB}$ throughout the entire sampled spectral range from 1.3 to $1.75 \mu \mathrm{m}$. This loss value is roughly five times lower than that achieved previously [2], mainly due to the use of a coupler for beam combination rather than a splitter and the better mode matching to optical fibers. The splitting ratio is also flat and well centered on $3 \mathrm{~dB}$. The coupling ratio 


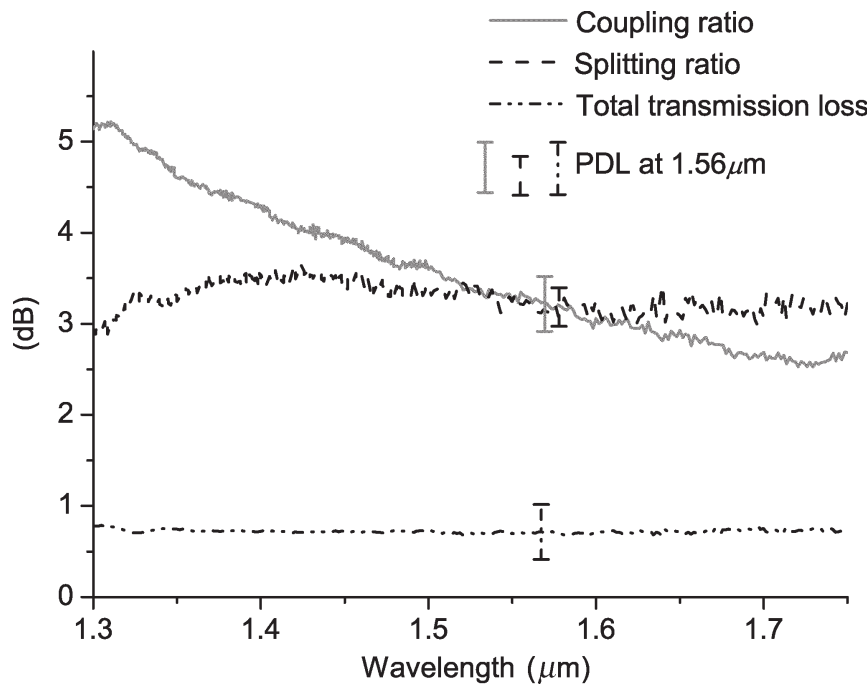

Fig. 3. Broadband variation of coupling ratio, splitting ratio, and total transmission loss of a beam combiner. The polarization dependence at $1.56 \mu \mathrm{m}$ is indicated with vertical bars.

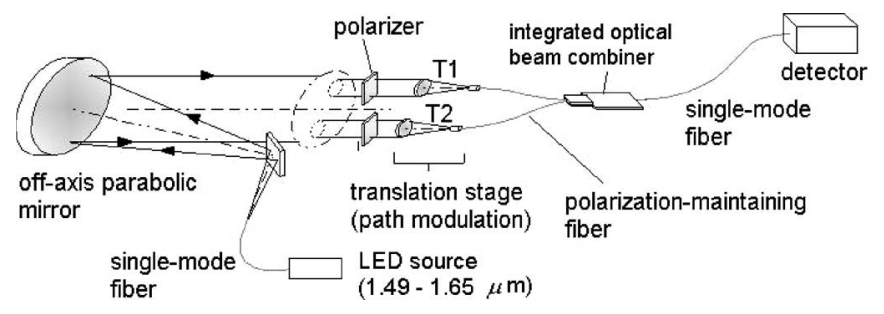

Fig. 4. Astronomical interferometric characterization test bed $(\mathrm{T} 1, \mathrm{~T} 2=$ telescopes).

exhibits a \pm 0.5 -dB variation from 1.5 to $1.75 \mu \mathrm{m}$. The \pm 0.5 -dB bandwidth of the coupler is most likely larger than this, since the measurement is truncated by an upper wavelength detection limit. In slightly different coupler designs, we have demonstrated a \pm 0.5 -dB bandwidth up to $0.45 \mu \mathrm{m}$ [9]. This bandwidth matches the best results obtained in more complex designs realized with other fabrication techniques and is roughly an order of magnitude larger than that of a standard symmetrical coupler.

\section{INTERFEROMETRIC VALIDATION}

Interferometric characterization was carried out on a dual telescope laboratory testbed (Fig. 4) with an optical configuration similar to that currently used in interferometric facilities at several astronomical observatories [3]. A collimated beam is produced using a fiber coupled to a light-emitting-diode (LED) source (1.49-1.65 $\mu \mathrm{m}$ output) and an off-axis parabolic mirror.

Two telescopes ( $\mathrm{T} 1$ and $\mathrm{T} 2$ ), placed along the beam path, feed the IO beam combiner input ports via polarizationmaintaining fibers. Telescope $\mathrm{T} 1$ is fixed while telescope $\mathrm{T} 2$ is mounted on a translation stage to realize a controllable opticalpath delay (OPD) with a range of $\pm 80 \mu \mathrm{m}$. In a practical working frame, the four output ports of the beam combiner are read simultaneously with a detector array so that flux fluctuations can be calibrated away by means of the photometric channels. In a laboratory environment, this is not necessary, so a singlemode fiber coupled to a single-pixel detector is used instead, and the signal intensity of each output is read subsequentially.

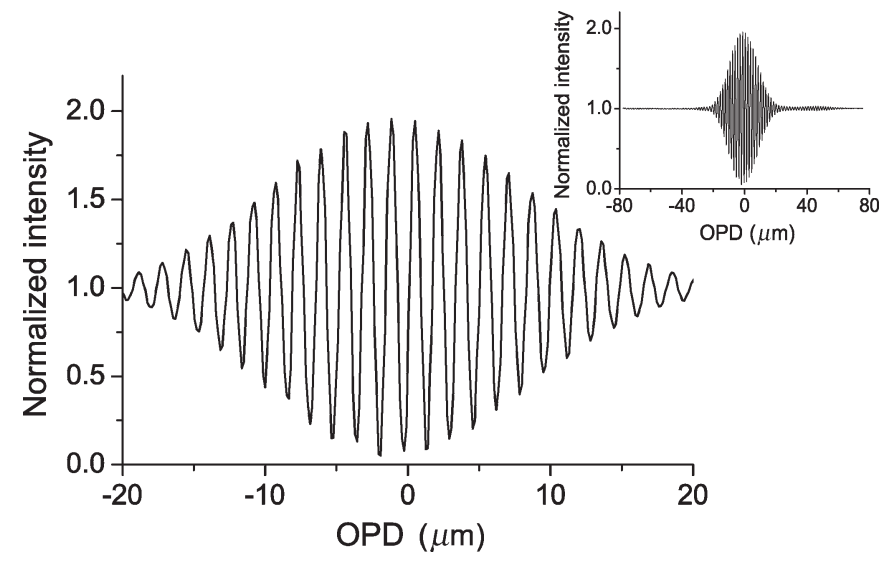

Fig. 5. Close-up view of a normalized interferogram measured with UVwritten dual-beam combiner, showing fringe contrast of $96 \%$. Inset: interferogram over the full $\pm 80 \mu \mathrm{m}$ OPD scan range.

A measurement sequence on one of the interferometric output ports consists of several OPD scans: 1) acquisition of interferometric signal (inputs $\mathrm{A}$ and $\mathrm{B}$ are fed, output $\mathrm{D}$ or $\mathrm{E}$ is read); 2) acquisition of photometric signal from input $A$ (B is cut off); 3) acquisition of photometric signal from input $B$ (A is cut off); and 4) acquisition of background signal (A and B are cut off). A polarizer is placed in front of both telescopes to improve the fringe contrast and stability by allowing the injection of linearly polarized light along the fiber + combiner fast or slow axis.

Two components with near-3-dB coupling ratio were selected for interferometric validation. A typical normalized interferogram is presented in Fig. 5, showing that the fringe contrast (visibility) is very high - typically $96 \%-99 \%$ for both components. This is better than the $92 \%$ visibility obtained in a previous realization with IO technology [2]. The fringe contrast decreases for nonzero OPD because of the limited temporal coherence of the source and the envelope of the fringe pattern is related to the spectral distribution of the LED source by a Fourier transform. The fringe contrast is ideally given by $2 \sqrt{r} /(1+r)$, where $r$ is the ratio between the fluxes upon recombination. Calculations of the fringe contrast using measured spectral variations of coupling and splitting ratio show good agreement with the measured values at $1.49-1.65 \mu \mathrm{m}$. For a lowest acceptable fringe contrast of $90 \%$, these calculations suggest that the beam combiner is functional throughout the entire single-mode regime (i.e., down to $\sim 1.3 \mu \mathrm{m}$ ), while the upper limit is undetermined due to our detection limit at $1.75 \mu \mathrm{m}$. By a small design change, the coupler spectral response curve and the single-mode wavelength range can be shifted $\sim 0.2-0.3 \mu \mathrm{m}$ to lower wavelengths, and then, the beam combiner could cover two astronomical bands, i.e., the J- and H-bands ranging from 1-1.8 $\mu \mathrm{m}$. Optical interferometry is highly desirable for observations in the near-infrared (up to the L-band at $3.75 \mu \mathrm{m}$ ) as well as in the midinfrared (e.g., N-band at $10 \mu \mathrm{m}$ ), where atmospheric fluctuations are less severe. Chalcogenide fibers guiding at $10 \mu \mathrm{m}$ have been studied for astronomical applications [15], while a recent experiment at the Keck telescopes [16] showed the possibility to use fluoride fibers to extend the interferometer capability up to $2.2 \mu \mathrm{m}$. On the other hand, direct UV writing has been demonstrated 


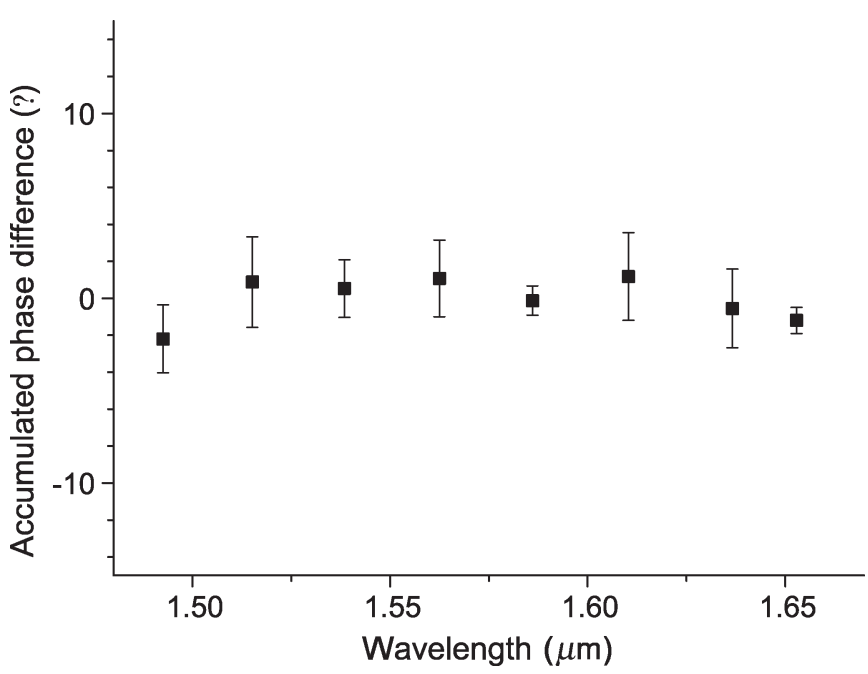

Fig. 6. Accumulated phase difference between interferometric arms of IO combiner versus wavelength. Error bars illustrate the standard deviation derived from multiple measurements.

both in chalcogenide [17] and fluoride glasses [18]. Therefore, it is possible, in principle, to reproduce our UV-written IO beam combiner in a new material with wider wavelengthoperation range. The main challenge will be the development of glass compositions that combine excellent transparency at the wavelengths of interest with a sufficient photosensitivity, whereas little work is likely to be required in terms of the UV-writing design and methods.

In general, differential chromatic dispersion between the interferometric paths, due to a poor equalization of fiber lengths and dissimilarities of the waveguides in the two arms, will lead to an accumulated phase difference and a reduction of the instrumental response. Since an astronomical combiner is made of the ensemble fibers + combiner, it is important to reduce the dispersion of both components. For the fiber case, care was taken to cut the two polarization-maintaining fibers at the same length, which was later measured to be identical to within $30 \mu \mathrm{m}$, hence yielding a negligible contribution to the phase difference [2]. The differential chromatic dispersion of the ensemble fiber + chip was then evaluated by means of Fourier transform spectroscopy in the range 1.49-1.65 $\mu \mathrm{m}$ (thus, covering part of the H-band). Two sets of measurements were performed with the polarization-maintaining fibers (Fig. 4) commutated to isolate any contribution of the beam combiner.

Several interferograms were acquired for each set and processed to evaluate the accumulated phase shift between the interferometric arms at each wavelength, as reported in Fig. 6. The experiment was repeated after a few days with another OPD translation stage to validate the data, and no difference between the two sequences could be observed within the measurement accuracy. The accumulated phase difference is at the setup detection limit of $\pm 2^{\circ}$ throughout the sampled wavelength range, with no discernable wavelength dependent trend. If any integrated-optics differential dispersion was present, we would have detected it as a curved phase in one of the two fiber injection configurations. Therefore, we can conclude that both the fiber pair and IO combiner have very limited differential dispersion. This result represents at least a fivefold reduction of the differential chromatic dispersion achieved with a similar design fabricated by ion exchange [2]. The achieved low onchip differential chromatic dispersion is due to 1) high homogeneity of the core layer fabricated by PECVD, yielding a low contribution of the material, and 2) high repeatability of UV-written waveguides, which ensures negligible differences between the propagation constants of the two arms over a wide spectrum.

A benefit of using single-mode waveguides for beam combination is that of modal filtering, which allows for transformation of wavefront phase corrugations into flux corrugations that can then be calibrated away using the photometric channels [2]. Modal filtering was verified by obtaining interferograms, where the input fibers had deliberately been misaligned by $3 \mu \mathrm{m}$, which did not produce any measurable change in the normalized fringe contrast.

Leaky modes excited at various locations on the beam combiner (inputs, branching points, etc.) can copropagate with the main circuit and later interfere with the photometric channels. To test for the presence of such parasitical interference, an OPD scan was performed while both inputs were fed, and the signal on a photometric channel was detected. There were no visible fringes on the direct signal, although a power-spectraldensity plot revealed a weak peak at the interferometric fringe frequency and a crosstalk level of $-40 \mathrm{~dB}$, which matches current astronomical requirements.

\section{CONCLUSION}

In this paper, the first realization by UV writing of an IO beam combiner for stellar interferometry has been presented. The device layout consists of two Y-branch splitters connected to a broadband-coupler section. Measurements show a total transmission loss of $\sim 0.7 \mathrm{~dB}$ and a polarization-dependent loss below $0.5 \mathrm{~dB}$. The interferometric performance, which was tested on a laboratory dual telescope testbed, demonstrates a fringe contrast of 96\%-99\%. The device can operate from 1.3 to at least $1.75 \mu \mathrm{m}$ with a fringe contrast above $90 \%$ while providing low crosstalk between photometric and interferometric output channels. The accumulated phase difference due to differential chromatic dispersion along the inteferometric paths is shown to be less than $\pm 2^{\circ}$ throughout the wavelength range $1.49-1.65 \mu \mathrm{m}$. The single-mode waveguides used in this design are shown to provide good modal filtering, thus allowing an improved calibration of wavefront corrugations. The overall device performance matches or exceeds that obtained previously reported with other IO fabrication techniques.

The performance level and fast prototyping ability of UV writing makes it a promising fabrication technique for demanding applications in stellar interferometry that require extensive optimization through a closed-loop design process. The development of new photosensitive materials sets a perspective for realization of UV-written devices with an extended operation to the midinfrared wavelengths. Moreover, since UV writing has already been used for demonstration of components with considerably more complex circuit layout than the simple dual-beam combiner presented here, an extension of the design to more input beams should be readily achievable. 


\section{REFERENCES}

[1] J. D. Monnier, "Optical interferometry in astronomy," Rep. Prog. Phys., vol. 66, no. 5, pp. 789-794, May 2003.

[2] P. Haguenauer, J.-P. Berger, K. Rousselet-Perraut, P. Kern, F. Malbet, I. Schanen-Duport, and P. Benech, "Integrated optics for astronomical interferometry. III. Validation of a planar optics two-telescope beam combiner," Appl. Opt., vol. 39, no. 13, pp. 2130-2139, May 2000.

[3] J.-P. Berger, P. Haguenauer, P. Kern, K. Perraut, F. Malbet, I. Schanen, M. Severi, R. Millan-Gabet, and W. Traub, "Integrated optics for astronomical interferometry. IV. First measurements of stars," Astron. Astrophys., vol. 376, no. 3, pp. L31-L34, 2001.

[4] M. Severi, P. Pouteau, P. Mottier, and P. Kern, "A waveguide interferometer for phase closure in astronomy," in Proc. Eur. Conf. Integr. Opt., 1999 , p. 279.

[5] M. Svalgaard, C. V. Poulsen, A. Bjarklev, and O. Poulsen, "Direct UV-writing of buried single-mode channel waveguides in Ge-doped silica films," Electron. Lett., vol. 30, no. 17, pp. 1401-1402, Aug. 1994.

[6] M. Svalgaard, K. Færch, and L. U. Andersen, "Variable optical attenuator fabricated by direct UV writing," J. Lightw. Technol., vol. 21, no. 9, pp. 2097-2103, Sep. 2003.

[7] M. Olivero and M. Svalgaard, "UV written $1 \times \mathrm{N}$ optical splitters," Opt. Express, vol. 14, no. 1, pp. 162-170, Jan. 2006.

[8] - "Fabrication of $2 \times 8$ power splitters in silica-on-silicon by the direct UV writing technique," IEEE Photon. Tech. Lett., vol. 18, no. 7, pp. 802-804, Apr. 2006.

[9] - "Direct UV-written broadband directional planar waveguide couplers," Opt. Express, vol. 13, no. 21, pp. 8390-8399, Oct. 2005.

[10] D. Zauner, K. Kulstad, J. Rathje, and M. Svalgaard, "Directly UV written silica-on-silicon planar waveguides with low insertion loss," Electron. Lett., vol. 34, no. 16, pp. 1582-1584, Aug. 1998.

[11] P. J. Lemaire, R. M. Atkins, V. Mizrahi, and W. A. Reed, "High pressure $\mathrm{H}_{2}$ loading as a technique for achieving ultrahigh UV photosensitivity and thermal sensitivity in $\mathrm{GeO}_{2}$ doped optical fibres," Electron. Lett., vol. 29, no. 13, pp. 1191-1193, Jun. 1993.

[12] T. Erdogan, V. Mizrahi, P. J. Lemarie, and D. Monroe, "Decay of ultraviolet light induced Bragg gratings," J. Appl. Phys., vol. 76, no. 1, pp. 73-80, Jul. 1994.

[13] J. B. LeBouquin, K. Rousselet-Perraut, P. Kern, F. Malbet, P. Haguenauer, P. Kervella, I. Schanen, J.-P. Berger, A. Delboulbé, B. Arezki, and M. Schöller, "First observations with an H-band integrated optics beam combiner at the VLTI," Astron. Astrophys., vol. 424, no. 2, pp. 719-726, Sep. 2004.

[14] K. Færch and M. Svalgaard, "Symmetrical waveguide devices fabricated by direct UV writing," IEEE Photon. Technol. Lett., vol. 14, no. 2, pp. 173-175, Feb. 2002

[15] P. Borde et al., "10 $\mu$ m-wavefront spatial filtering: First results with chalcogenide fibers," in Proc. SPIE-Astronomical Telescopes and Instrumentation, 2002, pp. 273-279.

[16] G. Perrin et al., "Interferometric coupling of the Keck telescopes with single-mode fibers," Science, vol. 311, no. 5758, p. 194, Jan. 2006.

[17] A. K. Mairaj, P. Hua, H. N. Rutt, and D. W. Hewak, "Fabrication and characterization of continuous wave direct UV $(\lambda=244 \mathrm{~nm})$ written channel waveguides in chalcogenide (Ga:La:S) glass," J. Lightw. Technol., vol. 20, no. 8, pp. 1578-1584, Aug. 2002.

[18] B. Boulard, L. Brilland, and H. Poignant, "UV writing of channel waveguides in erbium doped fluoride glass thin films," Electron. Lett., vol. 34, no. 3, pp. 267-268, Feb. 1998.

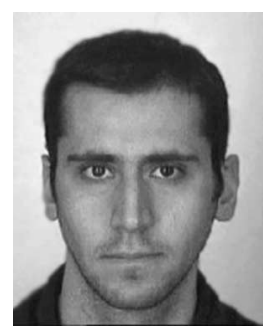

Massimo Olivero received the M.Sc. degree in electronic engineering and the Ph.D. degree in photonics from Politecnico di Torino, Torino, Italy, in 2002 and 2006, respectively.

Currently, he works as a postdoctoral researcher with the Politecnico di Torino. In 2004-2005, he was Visiting Researcher at the Research Center COM, Lyngby, Denmark, working on the development of integrated optical devices by direct UV writing. His current research interests are in fiber Bragg gratings, optical-fiber sensors, and UV-written integrated optical devices.

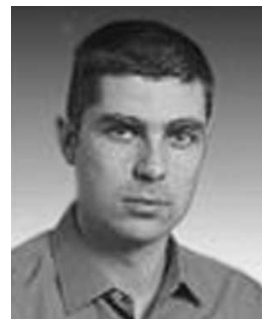

Mikael Svalgaard received the M.Sc. degree in physics from Copenhagen University, Copenhagen, Denmark, in 1993 and the Ph.D. degree in photonics from the Technical University of Denmark, Lyngby, in 1997.

He conducted research on optical wavelength standards at National Institute of Standards and Technology, Boulder, CO, in 1995 and 2003-2004. Since 2002, he has been an Associate Professor in nanophotonics with the Research Center COM, Technical University of Denmark, with focus on photonic crystals in III-V semiconductors for use in ultrafast lasers.

Laurent Jocou received the M.Sc. degree in optics from the Conservatoire des Arts et Métiers, Paris, France, in 2002.

From 1990 to 2000, he was with the Meudon Observatory, France, on the development of fiber links dedicated to spectrographs for astronomy. Since 2001, he has been working with Astrophysics Grenoble Observatory, Grenoble, France, where he is an engineer in instrumentation. His main interest is the development and implementation of interferometric combiner systems for astronomy using integrated optics components.

Jean-Philippe Berger received the Ph.D. degree in astrophysics from the University Joseph Fourier, Grenoble, France, in 1998.

He later joined the LEMO Laboratory at INP Grenoble as a French Space Agency Fellow to continue his research on the application of integrated-optics technology to astronomical interferometry. In 2000-2002, he joined Harvard Smithsonian Center for Astrophysics as a JPL/NASA Michelson Fellow. His current research as a Staff Astronomer with the Laboratoire d'Astrophysique de Grenoble involves the development of high angular resolution techniques to the study of star and planet formation. 